\section{RETROSPECTIVE ADDRESS}

\author{
OF THE
}

\section{PATHOLOGICAL SOCIETY OF READING. Read September 27th, I87 I.}

By WILLIAM ROY DS, L.R.C.P.Lond.

Mr. Presinent and Gentlemen, - I have not the presumption to undertake, without misgiving, the honourable task which your kindness has paid me the high compliment of imposing. To give an appropriate résumé of the proceedings of your Society for the past year, to review your papers and discussions, and fairly to recriticise your criticisms of the various subjects which have been brought before you, would require much greater knowledge and experience than I, a young member of your Society, can pretend to; and when I look back upon the long list of the names of those who have ably performed the duty which now devolves upon me of delivering the retrospective address to this the oldest Pathological Society in England, I feel that I must crave your indulgence whilst, to the best of my ability, I endeavour, by recapitulating the work of the past session, to perform the task which shall add my name to the roll.

The session I 870-7I has been in many respects a successful one. The meetings have been well-I believe, unusually well-attended, and the papers and discussions have not lacked interest; although I am struck, on looking through the list, at the scarcity of subjects having an interest purely medical, and venture to think that more of our time might profitably be devoted to medicine in the more limited application of the term, and especially in endeavouring to aid in filling up the "greatest gap" in the science, namely, that to be found "in its supreme and final stage", "the stage of therapeutics".

The variety of the past session has not been great, and I think I may best adopt the somewhat crude classification of medical, surgical, and obstetric subjects, and shall then have a few cases or papers which do not aptly fall under any of these heads, and to which I ruust refer as miscellaneous.

I. Medical Cases. - Cancer of the Brain. - Dr. Reid read the follow. ing interesting case, illustrated by the specimen which he presented. The patient was a female, aged 34. In May 1870 she underwent, at the hands of $\mathrm{Mr}$. Weeden Cooke, an operation for the removal of a cancerous tumour of the breast. The wound healed, and all seemingly went well with her until the beginning of August, when she was suddenly seized with loss of power in the right side and defect of speech. These symptoms passed off, but recurred with violent headache at the beginning of September. Another remission took place, leaving, however, impairment of speech, and persistent dull headache. In the beginning of October she suffered another attack of hemiplegia with aphasia. The symptoms became more and more intensified, the right arm ultimately remaining in a state of rigid flexion, and she died on December 2nd. The periods at which marked aggravation of the symptoms was noticed in each instance immediately preceded the appearance of the catamenia. On examining the brain after death, two deposits of cancerous matter were found in the surface and in the substance of the left cerebral hemisphere; each was about the size of a pigeon's egg, and the anterior one occupied the third left frontal convolution. The case thus formed an interesting addition to the rule of the production of aphasia by a lesion so situated. It is said to be rare to find more than one adventitious deposit in the brain; and where more than one exist, they are usually symmetrical. In this case it was not so. The nature of the disease had been diagnosed by Mr. May, and the situation of the deposit had been correctly predicted.

Aneurism of the Aorta: Tracheotomy. - The next case is one usually classed as a medical case, but in which surgical treatment was adopted unfortunately too late to be of much avail in prolonging life. A paper was read by Mr. Crisp on a case of aneurism of the aorta. H. D., aged 39, an officer's groom, consulted Mr. Crisp for an illness, which he stated to be of some weeks' duration, and to have commenced with cold, difficulty of breathing, alteration of voice, and cough. At the time when Mr. Crisp saw him he was in a very depressed and nervous state, unable to speak beyond a whisper; his voice, cough, and breathing, were markedly laryngeal. The left side of the thorax, anteriorly and superiorly, was more prominent than the right. There were deficiency of respiratory movements, and increased præcordial dulness. The breath-sounds were especially deficient, posteriorly and superiorly, on the left side. From the symptoms, Mr. Crisp judged that an intrathoracic tumour, probably an aneurism, existed. The same evening the patient was seized with a sudden and violent attack of spasmodic breath ing. This passed off, to recur several times within the next forty hours, the last attack leaving him in a state of utter exhaustion, perfectly unconscious, with his face pale, relaxed, and sweating; pulse undetectable; heart-sounds inaudible; respiration quite arrested. His friends considered that death had actually taken place. Tracheotomy was now rapidly performed, and artificial respiration was resorted to with the result of inducing respiratory efforts, and some return of the heart's action and of consciousness. The hopes thus raised of temporary relief being afforded were not, however, to be realised, as death ensued in about ten minutes from asthenia. The necropsy showed some chronic thickening about the cartilages of the larynx, and a diseased state of the aorta, and, at the termination of the transverse portion and the descending portion, an aneurism of the size of a goose's egg. The recurrent laryngeal nerve was in close contact with, and firmly adherent to, the tumour, and appeared to be somewhat hypertrophied. In this case death appeared finally to result from the exhaustion produced by the repeated attacks of laryngeal spasm, caused by the pressure of the aneurism on the recurrent laryngeal nerve. Death from asphyxia was obviated by the admission of air by an artificial opening; and the partial success of the operation would give encouragement for its earlier performance in any future case. The opinion of the Society was, that in a clear case the operation ought not to be deferred after the occurrence of the second attack of any violent spasm. One case occurred at the Winchester Hospital, in which the operation was resorted to with temporary relief.

Apoplexy.-Dr. Wells presented the left hemisphere of the brain of a woman, aged 57 , who was admitted into the hospital unconscious and with paralysis of the right side. She lived forty-two hours after admission. The left ventricle was found distended and broken up by effused blood. An interesting point in the history of the case was, that two of her brothers had also died of sanguineous apoplexy.

Perforation of the Stomach--Dr. Wells also read notes of a case of perforation of the stomach in a female, aged 59, whom he saw but once, and whose death occurred suddenly. An aperture of the size of a fourpenny piece was found in the upper curvature of the stomach near the cardiac end. Close upon its margin lay a lump of thickened tissue of the size of a pigeon's egg, apparently cancerous. The points of interest he stated to be as follows. First, although it was evident at the time of his visit that the patient was suffering from severe peritonitis, there was no indication that perforation existed. She was lying on her left side without any appearance of collapse, the pulse not being above roo. Death was sudden and unexpected. He supposed that the aperture had been closed by contiguous parts; and that, on its suddenly opening, the discharge of the contents into the cavity of the peritoneum occasioned a sudden shock to the system, in which she rapidly sank. Secondly, he noticed the relationship of the perforation to the mass of indurated tissue contiguous to it. The aperture did not look like the usual ulcerated opening from cancerous disease, but more like the smooth bevelled perforation which occurs from defective nutrition in chlorotic females.

Osseous Developments. - Mr. Harrinson read an elaborate paper on some osseous developments. The first part had reference to hydrocephalus and rickets, both of which, he maintained, contrary to the opinion of some authorities, might be congenital. He gave many points of distinction between the two diseases, and considered that the effusion of fluid into the cranial cavity in rickets resulted "not from any inordinate power of secretion, but from diminished resistance of the cranial parietes" due to the absorption, as quickly as formed, of the osseous tissue allowing dilatation, whereas in chronic hydrocephalus the distension depended upon compulsory dilatation, the parietes being normal. He gave the history of one case of congenital rickets which he had treated with suitable diet and a long course of phosphate of iron; marked improvement had already resulted, and he expressed great faith in the treatment, from which he hoped for ultimate restoration. Notwithstanding one distinguishing mark of hydrocephalus, which $\mathrm{Mr}$. Harrinson laid down, viz., "its tendency to get worse under any treatment", there seemed in the discussion which followed to be a strong opinion that the employment of iodide of potassium in what might, considering the tender age of the patients, be called heroic doses was likely, in some cases at least, to be productive of great benefit.-Another case of Mr. Harrinson's, illustrated by photographs, which he exhibited by means of the cosmoscope, was that of a girl who, at the age of I I years, began to have a swelling about the anterior third of the lower jaw on the right side, which in a few weeks increased to such a size as to produce great deformity. A London surgeon of eminence recommended its removal as an exostosis. A dentist advised 
delay. The latter course was adopted; and, as Mr. Harrinson had ventured to predict, the rest of the jaw "grew up" to this "anticipatory development", and, at the age of 16 , the swelling was scarcely noticeable. Although the result was thus good, Mr. Harrinson considered that the proper procedure would have been to extract an offending carious tooth, and scoop out the dental cavity, so removing the exciting cause of the excessive deposit.

Colloid Cancer of the Spleen.-Mr. Maurice exhibited a specimen from a case of colloid cancer of the spleen, involving also the peritoneum, in a man, aged 36 . It was remarkable for the large size and extent of the disease which existed without sooner fatally interfering with functions necessary to life. The mother and aunt of this patient had previously died of mammary cancer.

II. Surgical CASES. - Scirrhous Breast.-Mr. Maurice showed a remarkably large breast, which he had removed from a female, aged 40, for scirrhous disease. The question of operating was much debated, and the operation was at last undertaken with the hope that the patient's life might be prolonged, and the prospect afforded her of a more easy death than was probable were the disease left to take its course. The tumour, at the time of operating, was rapidly increasing and threatened speedily to involve the skin in ulceration; the axillary glands, too, were somewhat implicated. Whether either object of the operation was attained, is at least doubtful ; for although, when the paper was read, the patient was reported to have made rapid progress, to be very nearly well, and able to get about the house, before the wound had entirely healed the disease returned in it, and the patient speedily succumbed, and died within five months of the operation. Such cases are painful reminders of the shortcomings of the surgeon's art, and lead us to look anxiously, though scarcely even yet hopefully, for some means, other than surgical, whereby we may more successfully grapple with this hitherto unconquered foe.

Fungus Hamatodes. - Mr. Maurice described a case of fungus hæma. todes, for which he had performed amputation just above the knee. H. T. R., aged 54, but looking older, was admitted into the Royal Berkshire Hospital on Nov. Igth. He had a large inflamed swelling over the lower third of the left leg; on the anterior surface a small slough was protruding, about the size of a filbert. He stated that about two years ago he began to find pain in his leg at the seat of an old fracture (which occurred thirty years previously). Twelve months ago it began to swell; but nine weeks since the swelling became more persistent and was afterwards lanced, but there was not much discharge. After admission, another incision was made at the most prominent part of the swelling, and about two drachms of matter were evacuated. The lower end of the fibula was found to be diseased. Soon afterwards large unhealthy fungoid granulations appeared, which bled freely. These continued to increase in size, and the lymphatics of the leg became somewhat inflamed. On the $8 \mathrm{th}$ of December the limb was amputated just above the knee. The patient made a somewhat tedious recovery towards a tolerable state of health, and was discharged from the hospital. His recovery was never complete, and he died within a few months at a distance from Reading. The cause of his death was unknown. His life was probably prolonged and his death rendered more easy by relieving him of what, had its progress been unarrested, must have been a foul and unendurable incumbrance.

Excision of the Knee.-Mr. Moxhay reported a most interesting series of eight cases of excision of the knee, which he had performed in the Royal Berkshire Hospital. These have been published in the Midico-Chirurgical Transactions, and show the excellent result of seven recoveries, but one amputation (and that in a patient whose age and constitution rendered the operation almost a forlorn hope), and no deaths. These cases may surely tend to help the operation in winning its way to the entire acceptance of medical men, a result which, the author of the paper thought, had yet to come to pass.

Resection of the Hip.-Mr. Moxhay read an interesting paper on a case of resection of the hip, which he had performed on one of his hospital patients, a lad aged 16 . The disease of the joint came on very insidiously either during an attack of fever, or during the convalescence, whilst he suffered from a troublesome bedsore, for which last he was admitted into the hospital on November 2nd, 1869. In October, 1870, Mr. Moxhay removed the portions of bone exhibited, consisting of the greater part of the articular surface of the head of the femur, which was found lying loose in the healthy acetabulum, and the neck and part of the great trochanter. The bone was completely carious in much of its extent, and was found resting on a roughened surface of the dorsum ilii. In January, $187 \mathrm{I}$, union had taken place so firmly as to allow his being pushed up in bed by pressure on his heel. A note in May states that the wound was soundly healed, that he could flex, extend, and rotat the limb inwards, and to a slight extent outwards, and that he was able to bear the weight of the body on the toes.
In Mr. Moxhay's remarks, referring to the union which had taken place, he says, "It is evidently not bony, and yet it is very strong. It is at the same time somewhat yielding, for he can move the limb very freely by voluntary effort. Of course it must be ligamentous union, and we can only thank the beneficence of nature for providing such a means of repair where firmness and mobility are so much required. It is only surpassed by the original construction of the natural joint. This union is wonderfully different from what takes place after excision of the knee-joint. There you have the firm knitting together of the bones, which renders the limb more liable to fracture either at the femur above, or the tibia below the seat of union."

Mr. Moxhay considers that the great difficulty in deciding on the advisability of operative interference in these cases, lies in the fact "that one cannot know beforehand how much disease there may be in the acetabulum and neighbouring bone"; and that, the disease being generally caries and not necrosis, the difficulty of being certain that you have gouged away the whole of the diseased bone, is a real one. One valuable means, however, of getting rid of the first named difficulty, lies in making an exploring cut and using the finger as a probe to ascertain the extent of the disease.

Whilst on the subject of hip-joint disease, I cannot refrain from noticing the method of treatment now being brought before the profession in England by Dr. Sayre, which promises to revolutionise the practice followed in this too common ailment. By a comparatively simple instrument he renders practicable what has heretofore been an unfulfilled desideratum; viz., the application of a sufficient amount of extension to the femur to prevent the painful and injurious contact of its head with the acetabulum, at the same time allowing the patient to make use of the limb for the purpose of taking exercise. Whether the disease be looked upon as one generally of local or of constitutional origin, there can be no doubt that the prolonged confinement required in the carrying out of the important indication referred to by the usual means of splints applied to the limb or of weights attached to it, has a tendency to protract the case.

Median Lithotomy.-Mr. G. May brought forward two cases of calculus, in which he had performed the operation of lithotomy according to Allarton's method. The first patient was a man aged 68, who presented decided evidence of kidney-disease, but on whom, in consequence of the severity of the symptoms produced, $\mathrm{Mr}$. May ventured to operate. The stone was a large one of phosphate of lime, with a lithic acid nucleus, and much difficulty was experienced in its removal. Although at first promising well, the case terminated fatally from collapse on the third day. The other patient was a youth of A small oxalate of lime calculus was readily removed on September 2nd, 1870. On the third day all the urine passed per urethram, and the patient appeared to be recovering rapidly; but on the seventh the wound reopened, apparently in consequence of the urethra being obstructed by tenacious mucus. It continued open for a week, and then gradually closed, and he was discharged cured three weeks after the operation. In Mr. May's remarks he characterised the operation selected in these cases as one unequalled for facility of execution and for the safety and success of the results. He further remarked that it is not, however, a brilliant operation ; for, to ensure success, it is important that the prostate be slowly and gently dilated, ur incontinence of urine may result. A method which he thought it would have been better to have followed in the first case is one recommended by M. Dolbeau, to dilate the prostate slowly by a six-bladed dilator, and if the stone exceed an acorn in size to crush it by the lithotrite. This operation has been followed by greater success than any yet known.

Mr. Maurice referred to a case in which he had performed the lateral operation. He acknowledged its greater difficulty, but quoted a high authority for the opinion that incontinence of urine was a much too frequent consequence of Allarton's operation. I find, however, that Dr. Little of New York, as the result of forty cases of median lithotomy lately published, gives, amongst other advantages, that the patient retains control over the bladder.

\section{[To be continued.]}

Testimonial to Robert Roberts, Esq.-On the 7 th instant, a public meeting was held in the Assembly Room, Blaenau, Festiniog, for the purpose of presenting Mr. Roberts, Surgeon to Oakley's Hospital and Festiniog Slate Quarries, with a testimonial, as a token of the regard in which he is held by the inhabitants, and in appreciation of his valuable services rendered as a medical practitioner. The testimonial consisted of a purse containing $£ 220$, together with an address, and a book, elegantly bound, containing the names of the subscribers from half-a-crown upwards. 